\title{
Recent results from NA62 and NA48
}

\author{
Riccardo Fantechi' ${ }^{1}$; e-mail: fantechi@cern.ch \\ ${ }^{1}$ INFN - Sezione di Pisa
}

\begin{abstract}
This paper reviews recent results form the NA62 and NA48 experiments at CERN. NA62 has searched for the $K \rightarrow \pi v \bar{v}$ with an in-flight decay technique. The analysis of $1.2 \cdot 10^{11}$ decays has found one event compatible with the Standard Model prediction, giving an upper limit for the branching ratio of $1.4 \cdot 10^{-9}(95 \% \mathrm{CL})$ in the background assumption. Prospects for improvements in the measurement are given. Results on the $K_{l 3}$ decay form factors from NA48/2 are presented as well as the first observation of the decay $K^{ \pm} \rightarrow \pi^{ \pm} \pi^{0} e^{+} e^{-}$and the measurement of its branching ratio from the same experiment.
\end{abstract}

\section{Introduction}

\subsection{The quest for $K^{+} \rightarrow \pi^{+} v \bar{v}$ and $K_{L} \rightarrow \pi^{0} v \bar{v}$}

The ultra rare decays $K \rightarrow \pi v \bar{v}$ are, among the many rare flavour changing neutral current $\mathrm{K}$ and $\mathrm{B}$ decays, a powerful tool to search for new physics through underlying mechanisms of flavour mixing. Several circumstances allow to compute the SM branching ratio to very high precision:

- the $O\left(G_{F}^{2}\right)$ electroweak amplitudes exhibit a power-like GIM mechanism

\footnotetext{
*for the NA62 Collaboration: R. Aliberti, F. Ambrosino, R. Ammendola, B. Angelucci, A. Antonelli, G. Anzivino, R. Arcidiacono, M. Barbanera, A. Biagioni, L. Bician, C. Biino, A. Bizzeti, T. Blazek, B. BlochDevaux, V. Bonaiuto, M. Boretto, M. Bragadireanu, D. Britton, F. Brizioli, M.B. Brunetti, D. Bryman, F. Bucci, T. Capussela, A. Ceccucci, P. Cenci, V. Cerny, C. Cerri, B. Checcucci, A. Conovaloff, P. Cooper, E. Cortina Gil, M. Corvino, F. Costantini, A. Cotta Ramusino, D. Coward, G. D’Agostini, J. Dainton, P. Dalpiaz, H. Danielsson, N. De Simone, D. Di Filippo, L. Di Lella, N. Doble, B. Dobrich, F. Duval, V. Duk, J. Engelfried, T. Enik, N. Estrada-Tristan, V. Falaleev, R. Fantechi, V. Fascianelli, L. Federici, S. Fedotov, A. Filippi, M. Fiorini, J. Fry, J. Fu, A. Fucci, L. Fulton, E. Gamberini, L. Gatignon, G. Georgiev, S. Ghinescu, A. Gianoli, M. Giorgi, S. Giudici, F. Gonnella, E. Goudzovski, C. Graham, R. Guida, E. Gushchin, F. Hahn, H. Heath, T. Husek, O. Hutanu, D. Hutchcroft, L. Iacobuzio, E. Iacopini, E. Imbergamo, B. Jenninger, K. Kampf, V. Kekelidze, S. Kholodenko, G. Khoriauli, A. Khotyantsev, A. Kleimenova, A. Korotkova, M. Koval, V. Kozhuharov, Z. Kucerova, Y. Kudenko, J. Kunze, V. Kurochka, V.Kurshetsov, G. Lanfranchi, G. Lamanna, G. Latino, P. Laycock, C. Lazzeroni, M. Lenti, G. Lehmann Miotto, E. Leonardi, P. Lichard, L. Litov, R. Lollini, D. Lomidze, A. Lonardo, P. Lubrano, M. Lupi, N. Lurkin, D. Madigozhin, I. Mannelli, G. Mannocchi, A. Mapelli, F. Marchetto, R. Marchevski, S. Martellotti, P. Massarotti, K. Massri, E. Maurice, M. Medvedeva, A. Mefodev, E. Menichetti, E. Migliore, E. Minucci, M. Mirra, M. Misheva, N. Molokanova, M. Moulson, S. Movchan, M. Napolitano, I. Neri, F. Newson, A. Norton, M. Noy, T. Numao, V. Obraztsov, A. Ostankov, S. Padolski, R. Page, V. Palladino, C. Parkinson, E. Pedreschi, M. Pepe, M. Perrin-Terrin, L. Peruzzo, P. Petrov, F. Petrucci, R. Piandani, M. Piccini, J. Pinzino, I. Polenkevich, L. Pontisso, Yu. Potrebenikov, D. Protopopescu, M. Raggi, A. Romano, P. Rubin, G. Ruggiero, V. Ryjov, A. Salamon, C. Santoni, G. Saracino, F. Sargeni, V. Semenov, A. Sergi, A. Shaikhiev, S. Shkarovskiy, D. Soldi, V. Sougonyaev, M. Sozzi, T. Spadaro, F. Spinella, A. Sturgess, J. Swallow, S. Trilov, P. Valente, B. Velghe, S. Venditti, P. Vicini, R. Volpe, M. Vormstein, H. Wahl, R. Wanke, B. Wrona, O. Yushchenko, M. Zamkovsky, A. Zinchenko.
} 
- the top-quark loops largely dominate the matrix element

- the sub-leading charm-quark contributions have been computed at NNLO order [1]

- the hadronic matrix element can be extracted from the branching ratio of the $K^{+} \rightarrow \pi^{0} e^{+} v$ decay, well known experimentally [2].

The current predictions for the branching ratios are [3]:

$$
\begin{aligned}
& B R\left(K^{+} \rightarrow \pi^{+} v \bar{v}\right)=(8.4 \pm 1.0) \times 10^{-11} \\
& B R\left(K_{L} \rightarrow \pi^{0} v \bar{v}\right)=(3.4 \pm 0.6) \times 10^{-11}
\end{aligned}
$$

The uncertanties are dominated by the experimental knowledge of the external inputs. These decays are sensitive to extensions of the SM, like for instance:

- MSSM analyses [6,7]

- Littlest Higgs with T-parity [8]

- Simplified Z and Z' [9]

- Randall-Sundrum models with protective custodial symmetry [10]

- LFU violation models [11]

Thanks to the SM suppression and existing constraints from $\mathrm{K}$ physics, variations of the $K \rightarrow \pi v \bar{v}$ BRs from the SM predictions induced by new physics at mass scales up to 100 $\mathrm{TeV} / c^{2}$ can be observed with a $10 \%$ precision.

The decay $K^{+} \rightarrow \pi^{+} v \bar{v}$ has been observed by the experiments E787 and E949 at the Brookhaven National Laboratory and the measured branching ratio is $1.73_{-1.05}^{+1.15} \times 10^{-10}$ [12]. A limit $B R\left(K_{L} \rightarrow \pi^{0} v \bar{v}\right)<2.6 \times 10^{-8}$ at $90 \% \mathrm{CL}$ has been published by the E391a Collaboration[13].

\subsection{Semileptonic kaon decays}

The $\mathrm{K}_{l 3}$ decay width in the absence of radiative corrections can be represented by the Dalitz plot density depending on $\mathrm{E}_{l}$ and $\mathrm{E}_{\pi}[14]$ and the vector and scalar form factors, $f_{+}(t)$ and $f_{0}(t)$. For $K_{e 3}$ decays only $f_{+}(t)$ will contribute. The following form factor parametrizations have been used in the analysis: the quadratic parametrization [15], the pole parametrization [16] and the dispersive parametrization [17]. An improvement on the precision of the form factors measurement contributes to reduce the uncertainty of $\left|V_{u s}\right|$.

\subsection{The decay $K^{ \pm} \rightarrow \pi^{ \pm} \pi^{0} e^{+} e^{-}$}

Radiative kaon decays, dominated by long-distance effects, could bring information on shortdistance processes considering specific modes in specific kinematical areas. The case of $K^{ \pm} \rightarrow \pi^{ \pm} \pi^{0} e^{+} e^{-}$is relevant as the virtual photon giving the $e^{+} e^{-}$pair can be emitted either by Inner Bremsstrahlung (IB) or by Direct Emission (DE). These components, as well as their interference, offer interesting short and long-distance observables which can be studied in a kinematical space including the non-zero $q^{2}=m_{e e}^{2}$ variable. Only few theoretical publications are available [18-20] and the decay has not yet been observed. The NA48/2 measurements of the magnetic and electric terms in the $K^{ \pm} \rightarrow \pi^{ \pm} \pi^{0} \gamma$ decay has been used in [18] to predict the branching ratio of the single components of the $K^{ \pm} \rightarrow \pi^{ \pm} \pi^{0} e^{+} e^{-}$decay. 


\section{The NA62 experiment at CERN}

NA62 aims to collect about $100 \mathrm{~K}^{+} \rightarrow \pi^{+} v \bar{v}$ decays in two years of running, to measure the branching ratio with $10 \%$ precision, with an acceptance of $\sim 10 \%$. This measurement requires a beam line providing at least $10^{13}$ kaon decays, integrated over the data taking period. In addition a background rejection factor $\mathrm{O}\left(10^{12}\right)$ is required, to extract the signal in the overwhelming abundance of the main $K^{+}$decay modes. Indeed $K^{+} \rightarrow \pi^{+} \pi^{0}(21 \%)$ and $K^{+} \rightarrow \mu^{+} v(63 \%)$ should be rejected using respectively photon veto detectors and muon identifiers. The use of a high energy kaon beam $(75 \mathrm{GeV} / c)$ has an advantage in the first case, because for $P_{\pi^{+}}<35 \mathrm{GeV} / c$ the energy of the photons from the $\pi^{0}$ is larger than $40 \mathrm{GeV}$ and the efficiency for photon rejection is very high. Additional particle ID is needed to eliminate other backgrounds and to identify the pa rent kaon inside the unseparated beam.

The beam line can provide the required intensity of about $3 \times 10^{12}$ protons/pulse at an energy of $75 \mathrm{GeV}$ with $1 \%$ momentum bite. The beam contains $\pi^{+}(70 \%)$, protons $(23 \%)$ and $K^{+}(6 \%)$. The rate seen by the detectors along the beam line, integrated over a surface of $12.5 \mathrm{~cm}^{2}$, is about $750 \mathrm{MHz}$. The decay region is defined to be a $80 \mathrm{~m}$ volume downstream of the last beam line element. The decay volume is evacuated down to $10^{-6} \mathrm{mbar}$, to minimize the multiple scattering of the decay products and the number of interactions of the beam with the residual gas. The rate downstream is given mainly by kaon decay products and is about $10 \mathrm{MHz}$.

The layout of the detector is shown in Fig.1; the description of the apparatus can be found in [21].

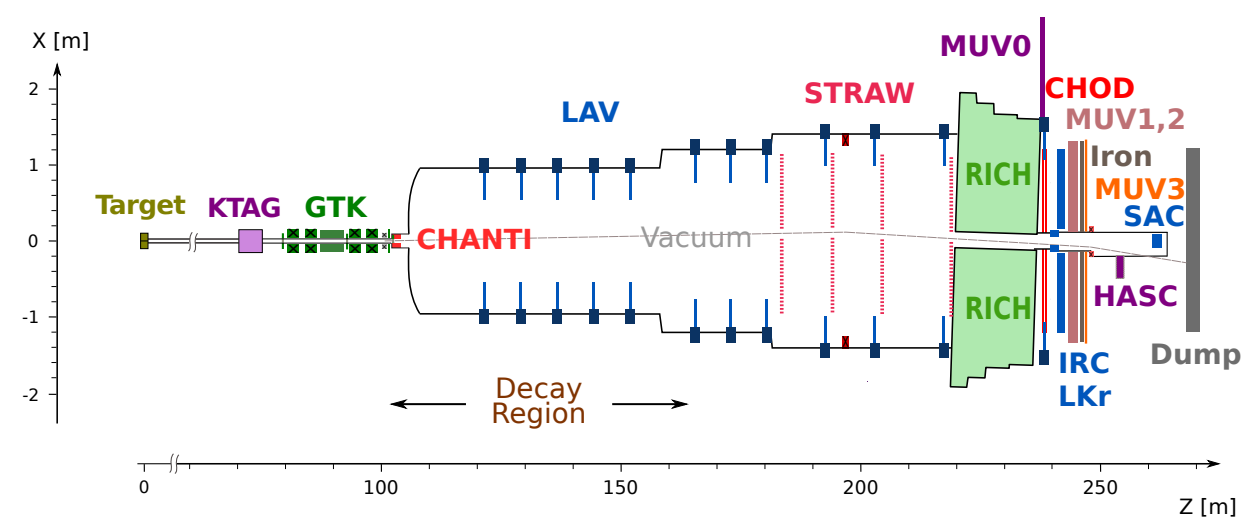

Figure 1. Horizontal view of the NA62 detector. The various labels refers to the detector elements.

\section{$3 K^{+} \rightarrow \pi^{+} v \bar{v}$ analysis strategy}

The analysis is based for the time being on a cut and count method, defining signal regions using the squared missing mass, $m_{m i s s}^{2}=\left(p_{K}-p_{\pi}\right)^{2}$ (Fig. 2), assuming the output particle is a $\pi^{+}$and computed for events with a single Straw track and for events with more than one track if no combination gives a vertex. Events with Straw track momentum larger than $35 \mathrm{GeV} / \mathrm{c}$ are rejected to better suppress the $K^{+} \rightarrow \pi^{+} \pi^{0}$, as lower track momentum implies higher $\pi^{0}$ energy, better detectable. A lower cut in track momentum of $15 \mathrm{GeV} / \mathrm{c}$ is applied, as in this case the pion is below the threshold in the RICH.

Events with hits in either the LAV, IRC, SAC and HASC in time with the $\pi^{+}$are rejected, as well as those with extra clusters in the LKr if they are more than $10 \mathrm{~cm}$ away from the 


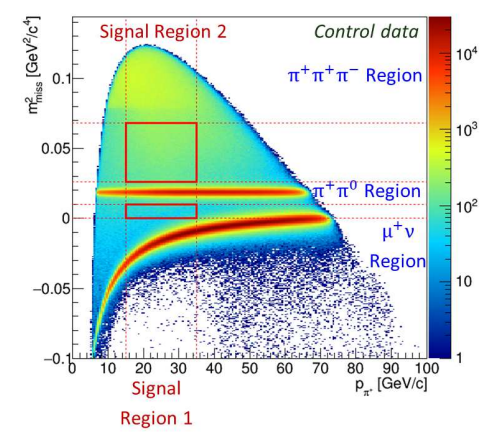

Figure 2. Kaon decays squared missing mass versus Straw track momentum.

Table 1. Expected signal and background events for the 2016 data

\begin{tabular}{ll}
\hline Process & Expected events in signal regions \\
\hline$K^{+} \rightarrow \pi^{+} v \bar{v}$ & $0.267 \pm 0.001_{\text {stat }} \pm 0.02_{\text {syst }} \pm 0.032_{\text {ext }}$ \\
\hline$K^{+} \rightarrow \pi^{+} \pi^{0}(\gamma) I B$ & $0.064 \pm 0.007_{\text {stat }} \pm 0.006_{\text {syst }}$ \\
$K^{+} \rightarrow \pi^{+} v(\gamma) I B$ & $0.020 \pm 0.003_{\text {stat }} \pm 0.003_{\text {syst }}$ \\
$K^{+} \rightarrow \pi^{+} \pi^{-} e^{+} v$ & $\left.0.018_{-0.021}^{+0.024}\right|_{\text {stat }} \pm 0.009_{\text {syst }}$ \\
$K^{+} \rightarrow \pi^{+} \pi^{+} \pi^{-}$ & $0.002 \pm 0.001_{\text {stat }} \pm 0.002_{\text {syst }}$ \\
Upstream Background & $\left.0.050_{-0.030}^{+0.090}\right|_{\text {stat }}$ \\
\hline Total Background & $0.15 \pm 0.09_{\text {stat }} \pm 0.01_{\text {syst }}$
\end{tabular}

impact point of the $\pi^{+}$. Muon background is rejected using the combined information from the calorimeters and the RICH.

The single event sensitivity (SES) is $S E S=\left(3.15 \pm 0.01_{\text {stat }} \pm 0.24_{\text {syst }}\right) \cdot 10^{-10}$, where the major contribution to the systematic error is due to the estimation of the loss due to random veto, which will improve in the future. Two signal regions are defined: Region I between the $K^{+} \rightarrow \mu^{+} v$ and $K^{+} \rightarrow \pi^{+} \pi^{0}$ peaks and Region II between the $K^{+} \rightarrow \pi^{+} \pi^{0}$ peak and the area populated by $K^{+} \rightarrow \pi^{+} \pi^{+} \pi^{-}$. The signal acceptance is $4 \%$, of which $1 \%$ in Region I and $3 \%$ in Region II.

Table 1 gives the number of expected signal and background events. One event is found in Region II (Fig. 3): the ring of the decay track in the RICH is shown in Fig. 4, consistent with a pion track.

In the assumption that the event is background, a 95\% upper limit could be placed

$$
B R\left(K^{+} \rightarrow \pi^{+} v \bar{v}\right)<14 \times 10^{-10}
$$

If the candidate is taken to be signal, the corresponding branching ratio at $68 \% \mathrm{CL}$ is

$$
B R\left(K^{+} \rightarrow \pi^{+} v \bar{v}\right)<28_{-23}^{+44} \times 10^{-11}
$$

This result is not competitive with the previous ones, but it shows that the in-flight technique works. NA62 has accumulated during 201720 times more statistics and it is continuing to take data in 2018, accumulating at the end of run about $20 \mathrm{SM}$ events. The collaboration is seeking approval to continue the data taking after LS2 to complete the measurement with a precision of $10 \%$. 


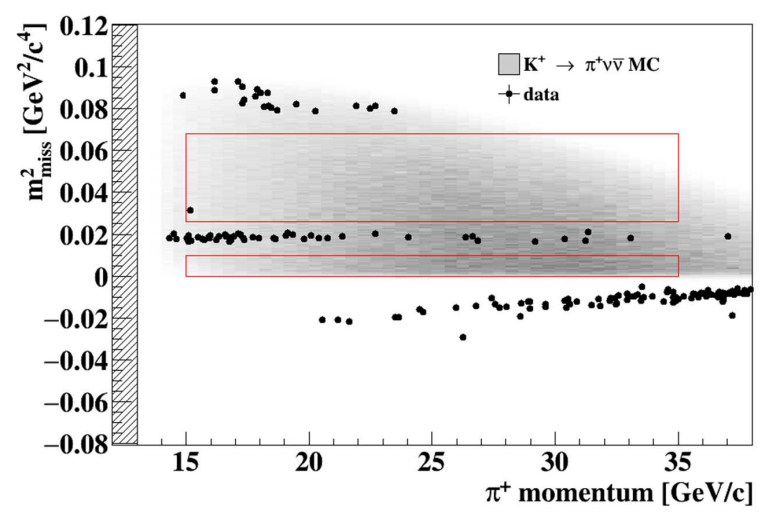

Figure 3. The events passing the analysis cuts, with the candidate in Region II

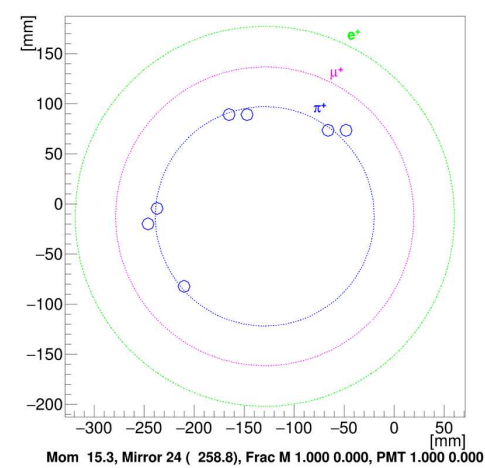

Figure 4. RICH hits for the candidate event

\section{Form factors of $K^{ \pm} \rightarrow \pi^{0} l^{ \pm} v$ decays}

The total number of events on the final samples of data taken with the NA48 detector [22] are $4.278 \times 10^{6} \mathrm{~K}_{e 3}$ and $2.907 \times 10^{6} \mathrm{~K}_{\mu 3}$.

The measurements of the form factors are obtained by minimizing the difference between data (background subtracted) and simulated Dalitz plots in $5 \times 5 \mathrm{MeV}^{2}$ bins. The semileptonic radiative Monte Carlo samples have been simulated with a specific generator [27].

NA48/2 is the first experiment measuring the form factors using both $K^{+}$and $K^{-}$. The $K_{\mu 3}$ result is dominated by the statistical error, the $K_{e 3}$ by the uncertainty on background. The $K_{\mu 3}$ and $K_{e 3}$ are in agreement within each other and the combined results are competitive with the current world average. To avoid partially correlated systematic uncertainties in the averaging of the $K_{\mu 3}$ and $K_{e 3}$ results, the complete analysis has been repeated considering the two decay modes as a unique data set, containing two Dalitz plots simultaneously fitted with a common set of form factor parameters. Fig. 5 shows the $39 \%$ confidence contours for the joint form factors using the quadratic parametrization, together with earlier measurements. 

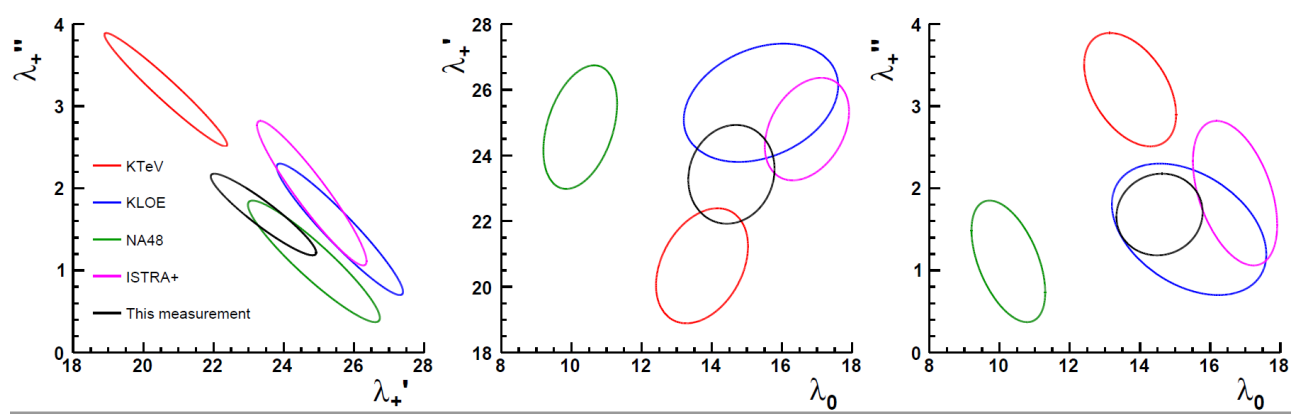

Figure 5. Confidence levels for the quadratic parametrization of the joint form factors. The ellipses correspond to $39 \% \mathrm{CL}$ ( one sigma contour).

\section{First observation of the decay $K^{ \pm} \rightarrow \pi^{ \pm} \pi^{0} e^{+} e^{-}$}

The main background contributions to the $K^{ \pm} \rightarrow \pi^{ \pm} \pi^{0} e^{+} e^{-}$signal are $K_{3 \pi D}$ where one photon is lost or merged with another particle and $K_{2 \pi D}$ where an extra photon combines with the Dalitz photon to mimic a $\pi^{0} \rightarrow \gamma \gamma$ decay. The first background is suppressed with a cut on the squared invariant mass of the $\pi^{+} \pi^{0}$ system to be greater than $0.12 \mathrm{GeV} / \mathrm{c}^{2}$. The second one with the condition that the two possible invariant masses $m_{e e \gamma}$ were more than $7 \mathrm{MeV} / \mathrm{c}^{2}$ away from the mass of the $\pi^{0}$. A total number of 5076 candidates has been selected. $\pi^{+} \pi_{D}^{0}$ is used as normalization with a total of 16.8 million candidates.

A detailed simulation based on GEANT3[24] has been used to compute the acceptances for the signal and the background, as well as for the determination of the background. Following the approach in [18], separate acceptances were computed for each component of the decay and then combined with the expected weights [18][23]. Several generators [25][26][27] have been used.

The most relevant background contributions to the signal are $K_{3 \pi D}$ with $159 \pm 8$ events and $K_{2 \pi D}$ with $130 \pm 24$ events (Fig. 6). For the normalization, $K_{\mu 3 D}$ contributes with $15182 \pm 173$ events, while $K_{e 3 D}$ with $10334 \pm 140$ events (Fig. 7).

The branching ratio is computed with

$$
B R\left(K^{ \pm} \rightarrow \pi^{ \pm} \pi^{0} e^{+} e^{-}\right)=B R_{n o r m} \cdot \frac{\left(N_{S}-N_{B S}\right) \cdot A_{N} \cdot \epsilon_{L 1 N} \cdot \epsilon_{L 2 N}}{\left(N_{N}-N_{B N}\right) \cdot A_{S} \cdot \epsilon_{L 1 S} \cdot \epsilon_{L 2 S}}
$$

The preliminary result is

$$
B R\left(K^{ \pm} \rightarrow \pi^{ \pm} \pi^{0} e^{+} e^{-}\right)=\left(4.22 \pm 0.06_{\text {stat }} \pm 0.04_{\text {syst }} \pm 0.13_{\text {ext }}\right) \times 10^{-6}
$$

Predictions from [18][23] are $4.183 \times 10^{-6}$ for IB only, $4.229 \times 10^{-6}$ including DE and INT terms and $4.145 \times 10^{-6}$ including also isospin breaking corrections, compatible with our result within the experimental errors.

\section{Conclusions}

NA62 has demonstrated the goodness of the in-flight technique to search for $K^{+} \rightarrow \pi^{+} v \bar{v}$ decays. With the analysis of the 2016 data, one candidate has been found with an expected background of 0.15 events. A $95 \%$ CL limit could be placed on the branching ratio as $B R\left(K^{+} \rightarrow \pi^{+} v \bar{v}\right)<14 \times 10^{-10}$.

Precise form factors for the semileptonic decays of $K^{ \pm}$have been obtained from the analysis of the NA48/2 data. 

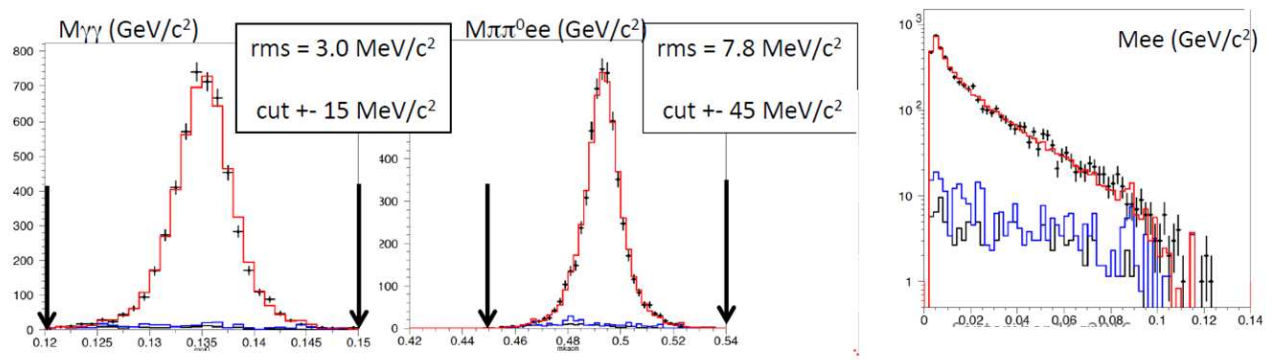

Figure 6. Data-MC comparison for the signal: reconstructed invariant masses for $\pi^{0}$ (left), for the kaon (center) and for $m_{e e}$ in log scale.
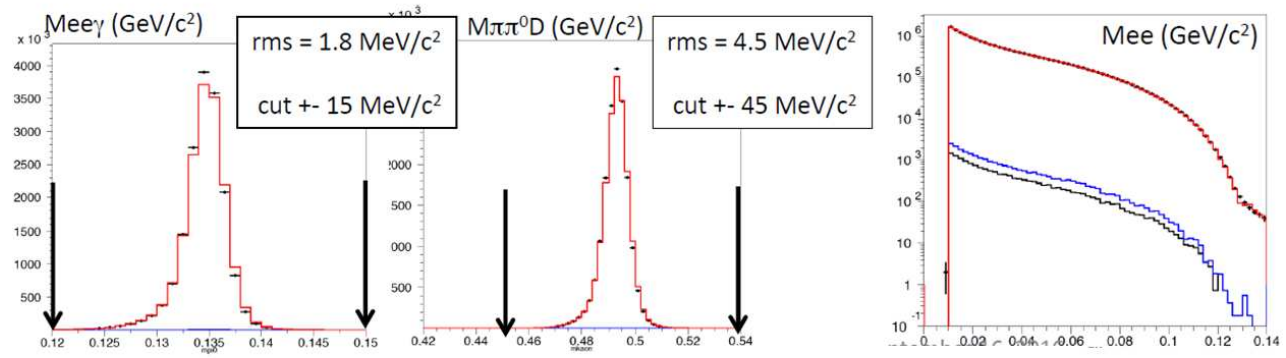

Figure 7. Normalization mode. Left: $M_{\pi^{0} D}$. Center: $M_{\pi \pi_{D}^{0}}$. Right: $m_{e e}$ spectrum in log scale.

NA48/2 reported the first observation of the decay $K^{ \pm} \rightarrow \pi^{ \pm} \pi^{0} e^{+} e^{-}$and measured a preliminary branching ratio, in agreement with theoretical predictions.

\section{References}

[1] A. J. Buras, M. Gorbahn, U. Haisch and U. Nierste, JHEP 0611 (2006) 002.

[2] C. Amsler et al., Phys. Lett. B667 (2008) 1.

[3] A.J. Buras, D. Buttazzo, J. Girrbach-Noe and R. Knegjens, JHEP 1511, 33 (2015).

[4] M. Blanke, A.J. Buras and S. Recksiegel, Eur. Phys. J. C76 (2016) 182.

[5] M. Blanke, A.J. Buras, B. Duiling, K. Gemmler and S. Gori, JHEP 0903 (2009) 108.

[6] T. Blazek and P. Matak, Int. J. Mod. Phys. A 29 (2014) no. 27, 1450162.

[7] G. Isidori, F. Mescia, P. Paradisi, C. Smith and S. Trine, JHEP 0608 (2006) 064.

[8] M. Blanke, A.J. Buras and S. Recksiegel, Eur. Phys. J. C76 (2016) 182.

[9] A.J. Buras, D. Buttazzo and R. Knegjens, JHEP 1511(2015) 166. 
[10] M. Blanke, A.J. Buras, B. Duiling, K. Gemmler and S. Gori, JHEP 0903 (2009) 108.

[11] M. Bordone, D. Buttazzo, G. Isidori and J. Monnard, Eur. Phys. J. C77 (2017) 618.

[12] S. Adler et al., Phys. Rev. D 79 (2009) 092004.

[13] J.K. Ahn et al., Phys. Rev. D 81 (2010) 072004.

[14] L.M. Chounet, J.M. Gaillard, M. Gaillard, Phys.Rept. 4, (1972) 199.

[15] C. Patrignani et al. (Particle Data Group), Chin. Phys. C 40, (2016) 100001.

[16] P. Lichard, Phys. Rev. D D 55, (1997) 5385.

[17] V. Bernard, M. Oertel, E. Passemar, J. Stern, Phys. Rev. D 80, (2009) 034034.

[18] L. Cappiello, O. Cata, G. D’Ambrosio and D. N. Gao, Eur. Phys. J. C 72 (2012) 1872.

[19] H. Pichl, Eur. Phys. J. C 20 (2001) 371.

[20] S. R. Gevorkyan and M. H. Misheva, Eur. Phys. J. C 74 (2014) 2860.

[21] E. Cortina Gil et al. [NA62 Collaboration], JINST 12 (2017) P05025.

[22] V. Fanti et al., Nucl.Instrum. Meth. A574, (2007) 433.

[23] L. Cappiello, O. Cata and G. D’Ambrosio, [arXiv:17012.10270 [hep-ph]].

[24] GEANT3 Detector Description \& Simulation Tool CERN Program Library W5013 (1994).

[25] E. Barberio and Z. Was, Comput. Phys. Commun. 79 (1994) 291.

[26] T. Husek, K .Kampf and J. Novotny, Phys. Rev. D 92 (2015) 054027.

[27] C. Gatti, Eur. Phys. J. C45 (2006) 417. 